\title{
Performance evaluation of compounding and directional beamforming techniques for carotid strain imaging using plane wave transmissions
}

Hansen, Hendrik H.G.; Stuart, Matthias Bo; Villagómez Hoyos, Carlos Armando; Jensen, Jørgen Arendt; de Korte, Chris L.

\section{Published in:}

Proceedings of IEEE International Ultrasonics Symposium

Link to article, DOI:

10.1109/ULTSYM.2014.0450

Publication date:

2014

Document Version

Early version, also known as pre-print

Link back to DTU Orbit

Citation (APA):

Hansen, H. H. G., Stuart, M. B., Villagómez Hoyos, C. A., Jensen, J. A., \& de Korte, C. L. (2014). Performance evaluation of compounding and directional beamforming techniques for carotid strain imaging using plane wave transmissions. In Proceedings of IEEE International Ultrasonics Symposium (pp. 1814-1817). IEEE. https://doi.org/10.1109/ULTSYM.2014.0450

\section{General rights}

Copyright and moral rights for the publications made accessible in the public portal are retained by the authors and/or other copyright owners and it is a condition of accessing publications that users recognise and abide by the legal requirements associated with these rights.

- Users may download and print one copy of any publication from the public portal for the purpose of private study or research.

- You may not further distribute the material or use it for any profit-making activity or commercial gain

- You may freely distribute the URL identifying the publication in the public portal 


\title{
Performance Evaluation of Compounding and Directional Beamforming Techniques for Carotid Strain Imaging using Plane Wave Transmissions
}

\author{
Hendrik H.G. Hansen ${ }^{1}$, Matthias B. Stuart ${ }^{2}$, Carlos A.Villagomez Hoyos ${ }^{2}$, Jørgen Arendt Jensen ${ }^{2}$, Chris L. de Korte ${ }^{1}$ \\ ${ }^{1}$ Medical UltraSound Imaging Centre (MUSIC), Department of Radiology and Nuclear Medicine, \\ Radboud University Nijmegen Medical Centre, Nijmegen, the Netherlands; \\ ${ }^{2}$ Center for Fast Ultrasound Imaging (CFU), Department of Electrical Engineering, \\ Technical University of Denmark, Lyngby, Denmark; \\ rik.hansen@radboudumc.nl
}

\begin{abstract}
Carotid strain imaging in 3D is not possible with conventional focused imaging, because the frame rate is too low. Plane wave ultrasound provides sufficiently high frame rates, albeit at the cost of image quality, especially in the off-axis direction due to the lack of focusing. Multiple techniques have been developed to cope with the low off-axis image quality when performing 2D (and in future 3D) motion estimation: cross correlation with directional beamforming (with or without RF (coherent) compounding) and displacement compounding. This study compares the precision of these techniques using linear array ultrasound data of a pulsating concentric homogeneous artery simulated using Field II. The transducer $\left(f_{c}=9 \mathrm{MHz}\right.$, pitch $=197.9 \mu \mathrm{m}, 192$ elements, $\left.f_{s}=180 \mathrm{MHz}\right)$ transmitted plane waves at 3 sequentially alternating angles $\left(0^{\circ},+\theta,-\theta\right)$ at a PRF of $2 \mathrm{kHz}$. Simulations were repeated for $\theta$ ranging from $1^{\circ}$ to $20^{\circ}$ with increments of $1^{\circ}$. Displacements were estimated for frame intervals of $1 / 15$ th $s$, tracked, and cumulated from diastole to systole using either displacement compounding, or directional beamforming optionally enhanced by RF compounding. 1D directional beamforming with $R F$ compounding and 2D displacement compounding with $\theta=\sim 20^{\circ}$ performed equally and best with a relative root-mean-squared error of $\sim 2 \%$ with respect to the analytical solution. The mean and standard deviation of the estimated motion direction for $2 \mathrm{D}$ displacement compounding with $\theta=20^{\circ}$ was $0.03^{\circ}+/-1.43^{\circ}$. Since displacement compounding requires no assumptions regarding the motion direction, this technique seems the best option for plane wave carotid strain imaging.
\end{abstract}

Keywords—beamsteering; carotid; compounding; directional beamforming; motion estimation; plane wave; strain imaging; ultrasound; vascular.

\section{INTRODUCTION}

Most people in Western society develop a disease called atherosclerosis when they age. Atherosclerosis is a systemic disease, which affects the geometry and mechanical properties of the arterial wall, due to the deposition of lipids, inflammatory cells, and other cellular debris inside the innermost layers of the arterial wall. Because of the change in geometry and mechanical properties, the deformation/strain pattern in the arterial wall caused by the pulsating blood also changes [1]. Intravascular studies in which ultrasound was used to estimate the radial strains in the coronary wall showed that it was possible to detect atherosclerotic plaques (localized regions of increased wall thickening) that were prone to rupture based on the strain pattern $[2,3]$. The detection of plaques prone to rupture is very important, because plaque rupture is regarded as cause for at least $60 \%$ of all strokes and myocardial infarctions [4].

Techniques for noninvasive estimation of arterial wall strains by ultrasound also exist [5-10]. However, most of these techniques allow strain estimation in 2D imaging planes only, because they are based on conventional focused ultrasound acquisition, which frame rates are too low for extension to 3D. Ultrasound imaging at high frame rates is possible when transmitting unfocussed plane waves. Instead of recording one image line at a time, a plane wave is transmitted over the full aperture of the transducer, which allows the acquisition of one image plane and even one image volume at a time. The lack of focusing comes at a cost: the signal-to-noise ratio is lower, and side-lobe levels are higher, which mainly affect the off-axis (lateral/elevational) image quality. As a consequence, motion estimation in these off-axis directions is also difficult. Since it is known that an artery moves in all 3 directions over the pressure cycle, motion estimation in these off-axis directions must be precise, otherwise the extension from 2D strain imaging to $3 \mathrm{D}$ strain imaging is useless. In the past decade, several methods have been developed to improve motion estimation in the off-axis directions: cross correlation with directional beamforming [11-13], displacement compounding [14-16], and compounding of radiofrequency (RF) data (coherent compounding) [17]. This study compares the performance of these methods for noninvasive vascular strain estimation by using simulations of a pulsating artery.

\section{MATERIALS}

Ultrasound RF element data of a homogeneous artery (Young's modulus, $\mathrm{E}=170 \mathrm{kPa}$ ) with a concentric lumen were simulated over a pressure cycle using Field II [18, 19]. The inner vessel wall radius at $0 \mathrm{mmHg}$ was $3.5 \mathrm{~mm}$ and the wall thickness was $1.5 \mathrm{~mm}$. The vessel was deformed according to a pressure curve of a 75 year old man [20]. The pressure curve is shown in Fig. 1A. More than 10 scatterers were placed per resolution cell $\left(350 / \mathrm{mm}^{3}\right)$ to ensure fully developed speckle was generated [21]. The radial positions of the scatterers $(R)$ in the wall were calculated for every $0.5 \mathrm{~ms}$ during the pressure 
cycle using the analytical plane strain equation for a homogeneous concentric tube [22]:

$$
R=r+\frac{a^{2} p}{E\left(b^{2}-a^{2}\right)}\left(\frac{b^{2}(1+v)}{r}+(1+v)(1-2 v) \cdot r\right)
$$

where $p$ is the instantaneous pressure, $r$ the radial position of a scatterer with respect to the lumen center at $0 \mathrm{mmHg}$ pressure, and $a$ and $b$ are the inner and outer radius at $0 \mathrm{mmHg}$ pressure, respectively. The Poisson's ratio ( $v$ ) was set to 0.495 .

Ultrasound data were simulated for a linear array transducer ( $f_{c}=9 \mathrm{MHz}$, pitch $=198 \mu \mathrm{m}, 192$ elements $)$ that transmitted plane waves at 3 sequentially alternating angles $\left(0^{\circ},+\theta,-\theta\right)$ at a PRF of $2 \mathrm{kHz}$, see Fig. 2. Simulations were repeated for $\theta$ ranging from $1^{\circ}$ to $20^{\circ}$ with increments of $1^{\circ}$. No apodization was applied in transmit and 128 elements were simultaneously active. The received element RF data were beamformed using delay-and-sum beamforming with an F-number of 0.875 . The maximum aperture width in receive was limited to 128 elements. Hamming apodization was applied in receive.

\section{METHODS}

\section{A. Displacement estimation: general settings}

All the investigated displacement estimation methods, which will be described in the next paragraphs, determine displacement estimates by calculating the location of the peak of normalized cross-correlation functions. These crosscorrelation functions are obtained by cross-correlating small kernels (template) of beamformed RF data from one ultrasound frame with larger kernels (search kernel) from another frame. To find the peak location at subsample level and, thus, to determine the motion at subsample level, parabolic interpolation was performed. Displacement vectors were estimated for 48 points equally distributed over two circles in the arterial wall, see Fig. 1B. The kernel sizes used for motion tracking were $0.43 \times 0.43 \mathrm{~mm}^{2}$ for the template, and $0.86 \mathrm{x}$ $0.86 \mathrm{~mm}^{2}$ for the search kernel (2D cross-correlation). Additionally, displacement estimation was performed using 1D kernels of $0.43 \mathrm{~mm}$ and $0.86 \mathrm{~mm}$ (1D cross-correlation). Points were beamformed for every $4 \mu \mathrm{m}$ (sampling rate $=180 \mathrm{MHz}$ ). The points in the vascular wall were tracked from the ultrasound frame at maximum pressure until the frame at minimum pressure using either displacement compounding, or
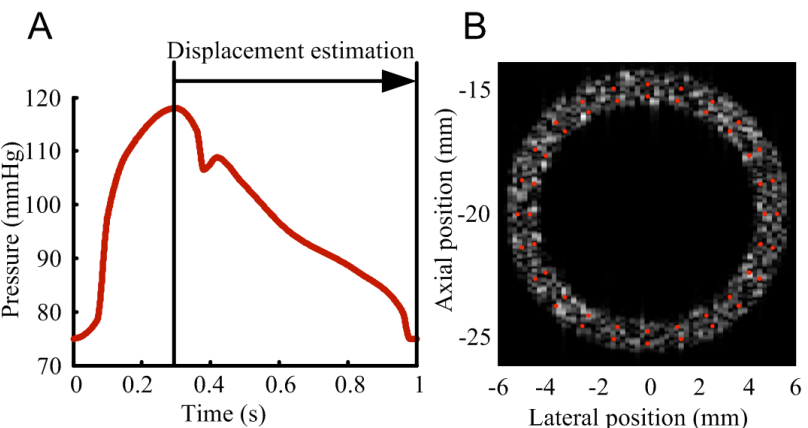

Fig. 1. A: Pressure curve of a 75-year old male that was used to deform a homogeneous concentric vessel. B: B-mode image of the artery at a pressure of $74 \mathrm{mmHg}$. Displacement estimation was performed from systole to diastole for the points in the arterial wall indicated by red dots. directional beamforming optionally enhanced by RF compounding. The displacements were estimated for frame intervals of $66 \mathrm{~ms}$. In case of compounding, subsequent acquisitions for the three angles were combined. A schematic overview of the different methods is presented in Fig. 2.

\section{B. Displacement compounding}

In displacement compounding, beamforming and crosscorrelation is performed in the direction of the steered plane waves [16]. The axial displacement estimate of the $0^{0}$ acquisition directly provides the vertical displacement. Projection of the axial displacements $u_{a x, \theta}$ and $u_{a x,-\theta}$ of the acquisition at $+\theta$ and $-\theta$ provides the horizontal displacement component:

$$
u_{h o r}=\frac{u_{a x, \theta}-u_{a x,-\theta}}{2 \sin \theta}
$$

From the horizontal and vertical displacement, the angle and magnitude of the $2 \mathrm{D}$ displacement is calculated. The strength of displacement compounding lies in the fact that the entire displacement vector is based on axial displacement estimates. Therefore, the reduced lateral image quality hardly affects the displacement estimation precision.

\section{Directional beamforming and RF compounding}

In directional beamforming, points are beamformed in the direction of motion, after which cross-correlation is carried out, hence, decorrelation due to out-of-plane motion is minimal [23]. In this study, the angle of motion is considered a given fact. Directional beamforming can be performed using $0^{0}$ data only, but it can also be enhanced by adding the RF data from the other two transmission angles (RF compounding) [10, 17].
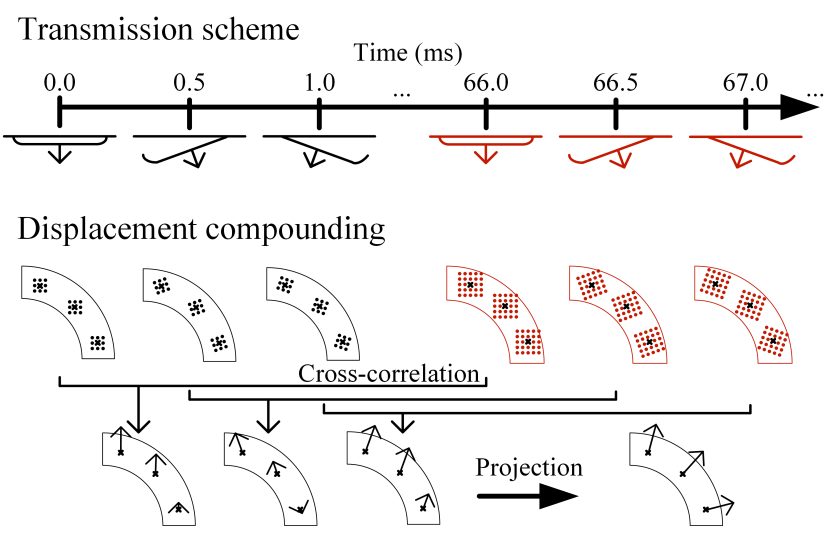

Directional beamforming with RF compounding

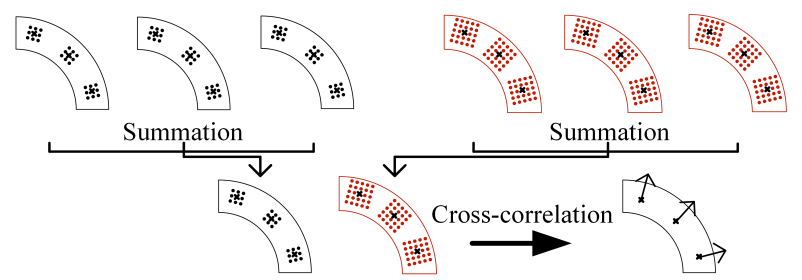

Fig. 2. Schematic overview of the transmission scheme, the displacement compounding method and the directional beamforming method enhanced by RF compounding. Displacement vectors have been enlarged for readability. 
In that case, beamforming is carried out at the same positions for all acquisition angles, after which the RF data are summed, and finally cross-correlated. The basic concept of RF compounding is the fact that it reduces the side-lobe levels, leading to an improvement in lateral image quality and improved displacement estimation performance.

\section{Analysis}

For each set of three acquisition angles, i.e. $0^{\circ},+\theta$, and $-\theta$ with $\theta$ ranging from $1^{\circ}$ to $20^{\circ}$ in steps of $1^{\circ}$, the precision of displacement compounding and directional beamforming (with $\mathrm{RF}$ compounding) was compared by calculating the relative root-mean-squared error (RMSE) of the estimated displacement magnitude with respect to the analytical solution (ground truth). In case of displacement compounding, the mean and standard deviation of the estimated motion angles with respect to the ground truth angles were also determined.

\section{RESULTS}

Fig. 3 shows the results for the various displacement estimation techniques as a function of the maximum plane wave angle. As can be observed the precision of $1 \mathrm{D}$ and $2 \mathrm{D}$ displacement compounding clearly improves as a function of the maximum plane wave angle. For 1D and 2D directional beamforming also an improvement is observed, although it is less pronounced. 1D directional beamforming outperforms $2 \mathrm{D}$ directional beamforming, whereas 2D displacement compounding outperforms $1 \mathrm{D}$ displacement compounding. 1D directional beamforming with RF compounding and $2 \mathrm{D}$ displacement compounding with $\theta=\sim 20^{\circ}$ provide the most precise displacement estimates with a relative RMSE of $\sim 2 \%$. The mean and standard deviation of the estimated motion direction was $0.03^{\circ}+/-1.43^{\circ}$ for 2D displacement compounding with $\theta=20^{\circ}$, see Fig. 4 .

\section{DisCUSSION}

The improvement in displacement compounding precision that was observed as a function of the maximum plane wave angle has been reported before for focused imaging [14]. For large plane wave angles, the angular axial estimates are more aligned with the horizontal displacement component, which enhances the precision of the projection. The improvements observed for RF compounding as a function of plane wave

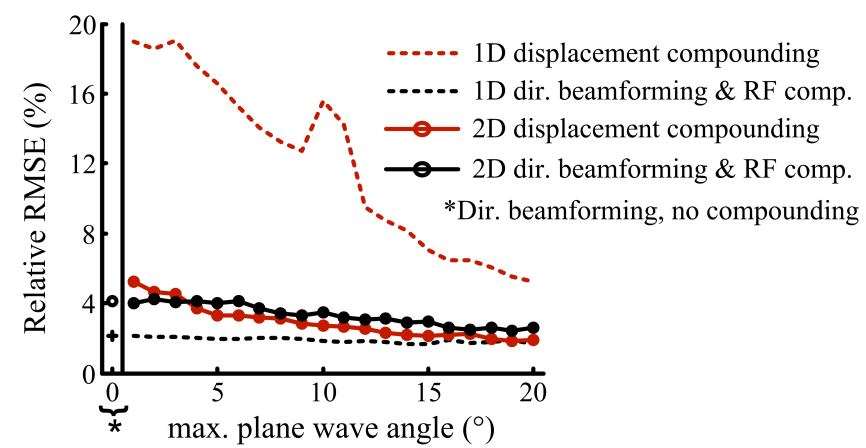

Fig. 3. Relative RMSE of the estimated displacement magnitudes obtained with the various beam forming methods as a function of the maximum plane wave angle.
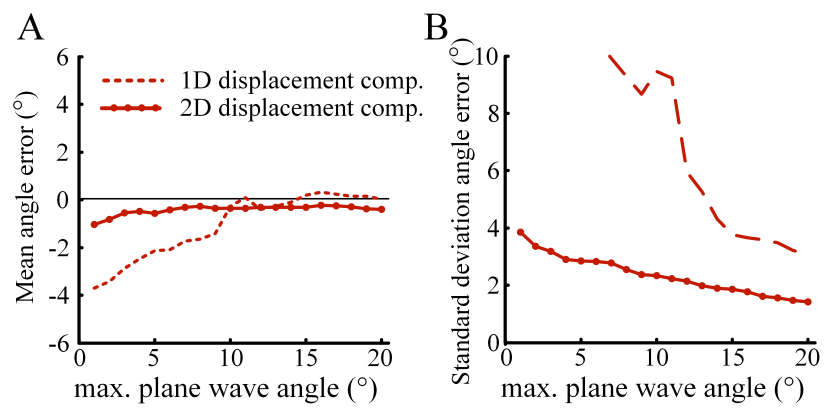

Fig. 4. A. Mean error and B. standard deviation of the estimated displacement angle for 1D and 2D displacement compounding as a function of the maximum plane wave angle.

angle are a result of the reduction in the effective horizontal beamwidth and the reduction of side-lobes.

The observation that 1D directional beamforming outperforms 2D directional beamforming is probably related to the fact that the displacement moves strains in the radial direction, which implies that more decorrelation occurs inside the kernel in case of 2D cross-correlation. The median crosscorrelation peak height for the 1D kernels was also slightly higher than that for 2D kernels, see Fig. 5. For displacement compounding 2D outperformed 1D. Thus, the same explanation does not hold for this method. The results for displacement compounding might be explained by the fact that the $1 \mathrm{D}$ cross-correlation was not performed in the direction of the displacement, and, therefore out-of-kernel displacement already caused de-correlations. This out-of-kernel displacement is probably partially corrected for in the 2D case, which explains why 2D displacement compounding is more precise. This explanation is again supported by Fig. 5: the median cross-correlation peak value is higher for $2 \mathrm{D}$ compared to $1 \mathrm{D}$.

In strain imaging usually spatial filtering is applied to remove outlying displacement values. In this paper this was not performed for two reasons: 1) to make a proper comparison between the methods without disturbance caused by nonlinear filtering, such as median filters, 2) because the displacement pixels were located at distances of $>1 \mathrm{~mm}$ which is much larger than the distances of $\sim 100 \mu \mathrm{m}$ normally used in strain imaging. In flow imaging usually also temporal filtering

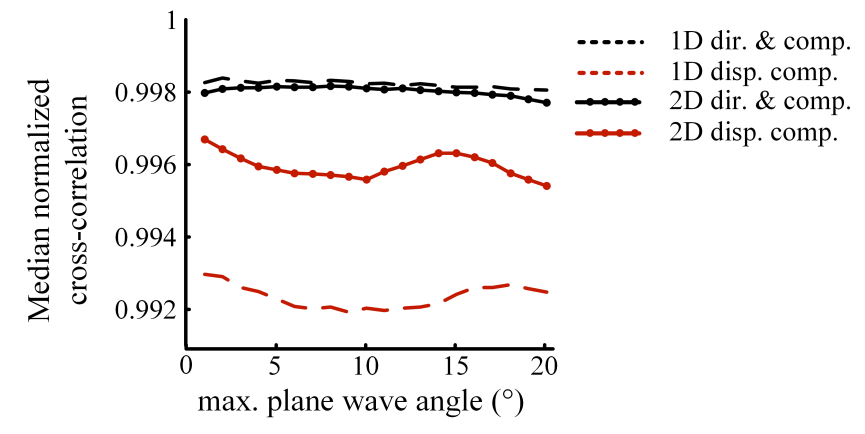

Fig. 5. Median normalized cross-correlation peak values for all four methods as a function of the maximum plane wave angle. As can be observed the highest cross-correlation values are found for $1 \mathrm{D}$ directional beamforming. 
is applied. This was also not performed in this study. However, for in vivo application in carotid arteries, both temporal and spatial filtering might be useful to further enhance the stability and precision of the displacement and strain estimation. Temporal filtering is expected to be possible, because of the relatively slow displacement in between two frames with an inter-frame time of 1/PRF. Moreover, for an imaging depth of $3 \mathrm{~cm}$, the PRF can even be further increased by a factor of ten.

In this study, the angle of displacement was considered a given fact for the directional beamforming method. However, when measuring in vivo, the angle of displacement needs to be determined. One way to determine the angle in vivo is by repeating the beamforming and cross-correlation in multiple directions. Since it can be expected that the signal correlates most in the direction of motion, the direction with the highest cross-correlation peak value is considered as the direction of motion. It was shown in flow imaging applications that this approach can provide angle estimates with a precision of 2 to $4^{\circ}[23,24]$. However, since the approach is computationally expensive and it might be more difficult to find the correct angle in a de-correlating vascular strain field, 2D displacement compounding seems the best option for carotid strain imaging. A multi-step approach in which the angles estimated by 2D displacement compounding are used as input for 1D directional beamforming is also a possibility, although this again increases the computational load.

\section{CONCLUSION}

2D displacement compounding using a $0^{\circ}$ plane wave transmission combined with two tilted plane wave transmissions at angles of $+20^{\circ}$ and $-20^{\circ}$ provides a precise estimate of the angle and magnitude of displacements without input of prior knowledge of the direction of motion. Therefore, for 2D (and in future 3D) ultrafast carotid strain imaging, displacement compounding seems a better option than directional beamforming with RF compounding.

\section{ACKNOWLEDGMENT}

This research is supported by the Dutch Technology Foundation STW (NKG 12122), which is part of the Netherlands Organization for Scientific Research (NWO), and which is partly funded by the Ministry of Economic Affairs.

\section{REFERENCES}

[1] Baldewsing, R.A., de Korte, C.L., Schaar, J.A., Mastik, F., and van der Steen, A.F.: 'Finite element modeling and intravascular ultrasound elastography of vulnerable plaques: parameter variation', Ultrasonics, 2004, vol. 42, (1-9), pp. 723-729

[2] Schaar, J.A., de Korte, C.L., Mastik, F., Strijder, C., Pasterkamp, G., Serruys, P.W., et al.: 'Vulnerable plaque detection with intravascular elastography: A sensitivity and specificity study', Circulation, 2001, vol. 104, (17), pp. II-459

[3] Schaar, J.A., de Korte, C.L., Mastik, F., Strijder, C., Pasterkamp, G., Serruys, P.W., et al.: 'Characterizing vulnerable plaque features by intravascular elastography', Circulation, 2003, vol. 108, (21), pp. 26362641

[4] Casscells, W., Naghavi, M., and Willerson, J.T.: 'Vulnerable atherosclerotic plaque: a multifocal disease', Circulation, 2003, vol. 107, (16), pp. 2072-2075
[5] Hansen, H.H.G., Lopata, R.G.P., and de Korte, C.L.: 'Noninvasive Carotid Strain Imaging Using Angular Compounding at Large Beam Steered Angles: Validation in Vessel Phantoms', IEEE Trans Med Imaging, 2009, vol. 28, (6), pp. 872-880

[6] Hansen, H.H.G., Lopata, R.G.P., Idzenga, T., and de Korte, C.L.: 'An Angular Compounding Technique Using Displacement Projection for Noninvasive Ultrasound Strain Imaging of Vessel Cross-Sections', Ultrasound in Medicine and biology, 2010, vol. 36, (11), pp. 1947-1956

[7] Hasegawa, H., and Kanai, H.: 'Phase-Sensitive Lateral Motion Estimator for Measurement of Artery-Wall Displacement-Phantom Study', Ieee Transactions on Ultrasonics Ferroelectrics and Frequency Control, 2009, vol. 56, (11), pp. 2450-2462

[8] Larsson, M., Kremer, F., Claus, P., Kuznetsova, T., Brodin, L.A., and D'Hooge, J.: 'Ultrasound-based radial and longitudinal strain estimation of the carotid artery: a feasibility study', IEEE Trans Ultrason Ferroelectr Freq Control, 2011, vol. 58, (10), pp. 2244-2251

[9] Maurice, R.L., Ohayon, J., Fretigny, Y., Bertrand, M., Soulez, G., and Cloutier, G.: 'Noninvasive vascular elastography: theoretical framework', IEEE Trans Med Imaging, 2004, vol. 23, (2), pp. 164-180

[10] Korukonda, S., Nayak, R., Carson, N., Schifitto, G., Dogra, V., and Doyley, M.M.: 'Noninvasive vascular elastography using plane-wave and sparse-array imaging', IEEE Trans Ultrason Ferroelectr Freq Control, 2013, vol. 60, (2), pp. 332-342

[11] Jensen, J.A.: 'Directional velocity estimation using focusing along the flow direction. I: Theory and simulation', IEEE Trans Ultrason Ferroelectr Freq Control, 2003, vol. 50, (7), pp. 857-872

[12] Jensen, J.A., and Bjerngaard, R.: 'Directional velocity estimation using focusing along the flow direction. II: Experimental investigation', IEEE Trans Ultrason Ferroelectr Freq Control, 2003, vol. 50, (7), pp. 873-880

[13] Jensen, J.A., and Nikolov, S.I.: 'Directional synthetic aperture flow imaging', IEEE Trans Ultrason Ferroelectr Freq Control, 2004, vol. 51, (9), pp. 1107-1118

[14] Hansen, H.H.G., Lopata, R.G.P., Idzenga, T., and de Korte, C.L.: 'Full 2D displacement vector and strain tensor estimation for superficial tissue using beam steered ultrasound imaging', Physics in Medicine and biology, 2010, vol. 5, (11), pp. 3201-3218

[15] Rao, M., and Varghese, T.: 'Spatial angular compounding for elastography without the incompressibility assumption', Ultrason Imaging, 2005, vol. 27, (4), pp. 256-270

[16] Hansen, H.H., Saris, A.E., Vaka, N.R., Nillesen, M.M., and de Korte, C.L.: 'Ultrafast vascular strain compounding using plane wave transmission', J Biomech, 2014, vol. 47, (4), pp. 815-823

[17] Tanter, M., Bercoff, J., Sandrin, L., and Fink, M.: 'Ultrafast compound imaging for 2-D motion vector estimation: application to transient elastography', IEEE Trans Ultrason Ferroelectr Freq Control, 2002, vol. 49, (10), pp. 1363-1374

[18] Jensen, J.A.: 'FIELD: A Program for Simulating Ultrasound Systems', Med Biol Eng Comput, 1996, vol. 34, supplement 1, part 1, pp. 351-353

[19] Jensen, J.A., and Svendsen, N.B.: 'Calculation of pressure fields from arbitrarily shaped, apodized, and excited ultrasound transducers', IEEE Trans Ultrason Ferroelectr Freq Control, 1992, vol. 39, (2), pp. 262-267

[20] Meinders, J.M., and Hoeks, A.P.: 'Simultaneous assessment of diameter and pressure waveforms in the carotid artery', Ultrasound Med Biol, 2004, vol. 30, (2), pp. 147-154

[21] Oosterveld, B.J., Thijssen, J.M., and Verhoef, W.A.: 'Texture of Bmode echograms: 3-D simulations and experiments of the effects of diffraction and scatterer density', Ultrason Imaging, 1985, vol. 7, (2), pp. 142-160

[22] de Korte, C.L., Céspedes, E.I., van der Steen, A.F.W., and Lancée, C.T.: 'Intravascular elasticity imaging using ultrasound: feasibility studies in phantoms', Ultrasound Med Biol, 1997, vol. 23, (5), pp. 735-746

[23] Jensen, J.A., and Oddershede, N.: 'Estimation of velocity vectors in synthetic aperture ultrasound imaging', IEEE Trans Med Imaging, 2006, vol. 25, (12), pp. 1637-1644

[24] Jensen, J.A.: 'Comparison of vector velocity imaging using directional beamforming and transverse oscillation for a convex array transducer'. Proc. SPIE Medical Imaging Conference, San Diego, USA 2014 